\title{
Loes Score: Clinical and Radiological Profile of 22 Patients of X-Linked Adrenoleukodystrophy: Case Series from a Single Center
}

\author{
Somesh Kumar ${ }^{1, \odot ~ H a s e e n a ~ S a i t ~}{ }^{1} \quad$ Sunil K. Polipalli ${ }^{1}$ \\ Sumit Pruthi ${ }^{3}$ Seema Kapoor ${ }^{1}$ \\ ${ }^{1}$ Division of Genetics and Metabolism, Department of Pediatrics, \\ Maulana Azad Medical College, New Delhi, India \\ ${ }^{2}$ Department of Radiodiagnosis, Maulana Azad Medical College, \\ New Delhi, India \\ ${ }^{3}$ Vanderbilt University Medical Centre, Nashville, Tennessee, United \\ States \\ Indian J Radiol Imaging 2021;31:383-390.
}

Gaurav S. Pradhan²

\begin{abstract}
Address for correspondence Seema Kapoor, MD, Division of Genetics and Metabolism, Department of Pediatrics, Maulana Azad Medical College, New Delhi, 110002, India (e-mail: drseemakapoor@gmail.com).
\end{abstract}

\begin{abstract}
Keywords

- adrenoleukodystrophy

- Loes score

- magnetic resonance imaging

- very long chain fatty acids

Introduction X-linked adrenoleukodystrophy (X-ALD) is a devastating disease with a wide spectrum of presentation ranging from asymptomatic to a rapidly progressive childhood cerebral form. The gene responsible for adrenoleukodystrophy is $A B C D 1$ gene, required for $\beta$ oxidation of fatty acids in various tissues. While biochemical and molecular techniques are available to confirm the diagnosis, brain magnetic resonance imaging (MRI) utilizing Loes score has been used for both prognosis and timely direction of hematopoietic stem cell therapy.

Materials and Methods During the study period of 2016 to 2020, 22 individuals including 19 individuals with features suggestive of X-ALD and 3 asymptomatic siblings were evaluated from a single center in North India. After biochemical and molecular confirmation of the disease, detailed clinical and radiological findings using MRI brain were documented. A radiological scoring pattern proposed by Loes was employed to identify the severity of the disorder.

Results The most common clinical presentations were visual difficulty and muscular weakness (58\%). All symptomatic individuals had classic neuroimaging findings in the form of hyperintensities involving the parieto-occipital area and splenium of corpus callosum. Severe involvement in the form of global atrophy was observed in $52.6 \%$ of individuals. Asymptomatic siblings also showed neurological involvement based on MRI with highest Loes score of 9 in one individual.

Conclusion This case series describes the clinical and radiological profile and employment of Loes score in individuals with X-ALD. Early identification of asymptomatic individuals by neuroimaging and use of Loes severity score for monitoring and disease progression will help in making therapeutic decisions in a timely manner.
\end{abstract}

published online July 28,2021
DOI https://doi.org/

$10.1055 / \mathrm{s}-0041-1734366$ ISSN 0971-3026
(C) 2021. Indian Radiological Association.

This is an open access article published by Thieme under the terms of the Creative Commons Attribution-NonDerivative-NonCommercial-License, permitting copying and reproduction so long as the original work is given appropriate credit. Contents may not be used for commercial purposes, or adapted, remixed, transformed or built upon. (https://creativecommons.org/licenses/by-nc-nd/4.0/). Thieme Medical and Scientific Publishers Private Ltd. A-12, Second Floor, Sector -2, NOIDA -201301, India 


\section{Introduction}

X-linked adrenoleukodystrophy (X-ALD) is a common peroxisomal disorder with a wide spectrum of clinical manifestations and classic imaging findings. The disease (\#OMIM 300100) is secondary to mutations in the ATP binding cassette subfamily D member 1 ( $A B C D 1)$ gene $^{1}$ that is responsible for $\beta$-oxidation of very long chain fatty acids (VLCFAs) in multiple tissues like the adrenal gland, central nervous system myelin, and Leydig cells of the testicles. The commonest phenotype of X-ALD is childhood cerebral adrenoleukodystrophy (CCALD) that presents between 4 and 10 years of age. Other types include late onset cerebral forms (CALD), adrenomyeloneuropathy, and Addison only presentation. The life-time risk of patients with mutation in the $A B C D 1$ gene to develop CCALD is $~ 60 \%$ and usually occurs between 5 and 12 years of age. ${ }^{2}$

The typical neuroimaging findings of deep white matter involvement in the parieto-occipital lobes and splenium of the corpus callosum generally prompt further confirmatory testing. Analysis of VLCFAs profile on dried blood spots is a good sensitive biomarker for X-ALD but not specific and hence requires molecular confirmation. However, one cannot underestimate the importance of neuroimaging as a screening and diagnostic tool especially in the presymptomatic stage where both behavior and cognition are normal. In cases where the involvement of the central nervous system is detected earlier, hematopoietic stem cell transplantation (HSCT) can be a best option to arrest the disease progression. Even in situations where an appropriate donor is unavailable, role of gene therapy is currently being investigated to halt the disease process. ${ }^{3}$ Hence, it is important to detect this disease as early as possible as these therapies can only be effective during a short therapeutic window. This knowledge has also largely driven the implementation of newborn screening for $\mathrm{X}$-ALD across some countries in the globe. ${ }^{4}$

Loes in 1994 developed an imaging-based scoring system for patients with X-ALD based on the neuroanatomical involvement and the presence or absence of total and/or global atrophy. This 34-point imaging-based severity scale was based on the location and extent of central nervous system involvement and presence of either focal or global atrophy. ${ }^{5}$ The score thus obtained helps in the prediction of disease course and selection of patients for HSCT. Reports on the clinical and imaging spectrum of patients with X-ALD from the Indian subcontinent are sparse. We hereby discuss the clinical and neuroimaging findings from 22 individuals with CCALD and CALD subtype of X-ALD seen at our center.

\section{Subjects and Methods}

The study was approved by the Institutional Ethical Committee for research on human subjects. Nineteen individuals presenting with symptoms suggestive of CCALD or CALD forms of X-ALD and three asymptomatic individuals who were identified on cascade screening of the male siblings of the family in question at the time of presentation were evaluated during the study period (2016-2020).
Clinical and neurological evaluation was performed in all patients. The parameters noted were age at manifestation of first symptom, age at presentation to the clinic, features suggesting adrenal dysfunction including presence of hyperpigmentation, neuroregression, seizures, sensory and behavioral disturbances, muscle weakness, difficulty in walking and visual, and hearing loss. Progression of symptoms in the affected individuals and onset of symptoms in asymptomatic siblings were monitored by 3 monthly follow-up.

Biochemical analysis was done on dried blood spots using Neobase kit 2.0 on an ABI 3200MD QTrap LCMSMS systems using flow injection technique. Primary metabolite assessed was lysophosphatidylcholine C26:0 (cutoff: $0.604 \mu \mathrm{mol} / 3.2 \mathrm{~mm}$ punch). The confirmation of the diagnosis was made by sequencing the $A B C D 1$ gene using a 24 capillary sequencer ( $A B I 3500 x L$ ). If sequencing did not identify a mutation, multiplex ligation-dependent probe amplification was performed for detecting deletions/duplications if any. For novel variants, bioinformatics analysis was performed using Polyphen, sorting tolerant from intolerant (SIFT), Mutation taster softwares and segregation studies were also performed to confirm the pathogenicity. Only those fulfilling the biochemical and molecular criteria were further assessed.

Magnetic resonance imaging (MRI) was obtained on a 3 tesla (Siemens, Munich, Germany; General Electric Medical Systems, Milwaukee, Wisconsin, United States; Philips Medical Systems, Cleveland, Ohio, United States) and for few MRIs that were performed outside the institute; images were obtained in a CD-ROM in DICOM format and reviewed by the single neuroradiologist to ensure no observer bias. The scoring system was obtained from the study by Loes et $\mathrm{al}^{5}$ with the assessment of abnormalities on T2-weighted, T1-weighted, and postcontrast images. Advanced neuroimaging parameters such as diffusion tensor imaging (DTI) or single voxel magnetic resonance spectroscopy were not available for majority of the patients and hence were not analyzed. Supratentorial white matter including corpus callosum and visual pathway, frontopontine and brain stem projection fibers, corticospinal tract, cerebellum, and basal ganglia regions were assessed for disease involvement. White matter was divided into three territories: anterior temporal, frontal, and parieto-occipital white matter (which includes the posterior temporal white matter lying posterior to the anterior margin of midbrain). The supratentorial white matter was also divided into periventricular, central, and subcortical divisions. These divisions were defined as being approximately equal in thickness to cortical gray matter. If involvement was unilateral for a specific location, a score of 0.5 was given. If involvement was doubtful for a specific location without evidence of any other abnormality, a score of 0.5 was given. Higher scores represent severe involvement of white matter. In our study, the presence of a brain lesion was given a Loes score of $\geq 0.5$. Focal atrophy was identified by a qualitative loss of brain parenchyma and global atrophy was assessed by both quantitative and qualitative measure. A scoring of one point was given for prominent subarachnoid space and the diameter of third ventricle between 5 and $10 \mathrm{~mm}$. Two points were allocated if the diameter of 
third ventricle was $>10 \mathrm{~mm}$. One point was allocated if the bifrontal horn to inner table ratio was more than $40 \%$. A ratio of more than $50 \%$ was given 2 points and a maximum of 3 points were given for higher ratios. Following calculation of Loes score, patients were grouped into multiple categories based on the severity of scores $(4,5-10>10)$. These categories were chosen as patients with scores $<9$ respond better to $\mathrm{HSCT}^{6}$ and early inclusion of individuals with score $\leq 4$ for HSCT was recently proposed.

Postcontrast sequences when available were analyzed for the presence of contrast enhancement of the lesions. Whenever present, the pattern of contrast enhancement was also recorded. Diffusion-weighted images (DWI) when available were analyzed and the findings were recorded. All these measurements were taken at a single time frame except in two familial cases, where HSCT was envisaged and the images were obtained twice at an interval of 9 and 12 months prior to the decision for HSCT. The summary of MRI severity scale proposed by Loes in presented in - Table 1.

Statistical analysis: Stata 11 software analysis was employed. Data on various outcome variables were presented as mean and standard deviation. Percentages were used wherever applicable.

\section{Results}

During the 5-year period (2016-2020), 22 cases from 19 families with a clinical and radiological phenotype

Table 1 MRI severity scale scoring (Loes et al ${ }^{5}$ )

\begin{tabular}{|c|c|}
\hline $\begin{array}{l}\text { Parieto-occipital white matter (max- } \\
\text { imum 4) }\end{array}$ & \multirow[t]{2}{*}{$\begin{array}{l}\text { Basal ganglia } \\
\text { (maximum 1) }\end{array}$} \\
\hline $\begin{array}{l}\text { Anterior temporal white matter } \\
\text { (maximum 4) }\end{array}$ & \\
\hline $\begin{array}{l}\text { Frontal white matter } \\
\text { (maximum 4) } \\
\text { - Periventricular } \\
\text { - Central } \\
\text { - Subcortical } \\
\text { - Local atrophy }\end{array}$ & $\begin{array}{l}\text { Visual pathway } \\
\text { (maximum 4) } \\
\text { - Optic radiation } \\
\text { - Meyer's loop } \\
\text { - Lateral geniculate } \\
\text { body } \\
\text { - Optic tract }\end{array}$ \\
\hline $\begin{array}{l}\text { Corpus callosum } \\
\text { (maximum 5) } \\
\text { - Splenium } \\
\text { - Genu } \\
\text { - Body } \\
\text { - Splenium atrophy } \\
\text { - Genu atrophy }\end{array}$ & $\begin{array}{l}\text { Auditory pathway } \\
\text { (maximum 4) } \\
\text { - Medial geniculate body } \\
\text { - Brachium of inferior } \\
\text { colliculus } \\
\text { - Lateral lemniscus } \\
\text { - Pons }\end{array}$ \\
\hline \multirow{2}{*}{$\begin{array}{l}\text { Global atrophy } \\
\text { (maximum 4) } \\
\text { - Mild } \\
\text { - Moderate } \\
\text { - Severe } \\
\text { - Brainstem }\end{array}$} & $\begin{array}{l}\text { Cerebellum } \\
\text { (maximum 2) } \\
\text { - White matter } \\
\text { - Atrophy }\end{array}$ \\
\hline & $\begin{array}{l}\text { Projection fibers } \\
\text { (maximum 2) } \\
\text { - Internal capsule } \\
\text { - Brain stem }\end{array}$ \\
\hline
\end{tabular}

Abbreviation: MRI, magnetic resonance imaging.

Each region is given a score of 0 for normal, 0.5 for unilateral involvement, and 1 for bilateral involvement or atrophy. The maximum score is 34 . suggestive of CCALD and late onset CALD forms of X-ALD were assessed. Among them, 10 had CCALD phenotype, 9 had CALD phenotype, and 3 individuals from 3 unrelated families were asymptomatic and were identified from cascade screening. Consanguinity was present in a single family. All of them had molecular confirmation of the diagnosis along with elevated VLCFA and C26:0 levels and significant findings on MRI.

The mean age of presentation of CCALD was 7.5 years (standard deviation [SD]: 1.8) and of CALD was 11.3 years (SD: 1.5). The clinical features of symptomatic individuals are depicted in - Table 2. Visual difficulty and muscular weakness were the most common symptoms (58\%). All symptomatic individuals had classic MRI picture of X-ALD in the form of hyperintensities involving the parieto-occipital area and splenium of corpus callosum. The other common areas of involvement were the visual and auditory pathway $(n=17$ [89.5\%] and $n=12$ [63\%]), respectively. Involvement of projection fibers of brain stem was observed in 13 symptomatic individuals (68.4\%) and that of frontotemporal area in 9 individuals (47.3\%). Global atrophy that was observed in 10 individuals (52.6\%) also had onset of disease in early childhood indicating the rapid progression of the disease ( $\mathbf{- T a b l e} \mathbf{3}$ ).

Loes severity score was calculated for each of these patients. The mean Loes score of symptomatic individuals was 13.7 (minimum score: 5, maximum score: 30). -Figs. 1 to 3 show representative MRIs of symptomatic and asymptomatic subjects with Loes score calculation. It was evident that asymptomatic individuals also had characteristic changes in MRI brain. While demyelination in the parieto-occipital region was observed in all three individuals, involvement of splenium of corpus callosum and visual pathway was observed in two of them and projection fibers of brain stem was involved in one subject. Two of these asymptomatic individuals had an initial Loes score of 0 and 1, but on follow-up after 6 and 9 months were found to have progression of lesions with Loes scores of 1 and 2. Even the third individual with a higher Loes score (score of 9) ( - Fig. 3) did not show any clinical symptoms. Loes score was also categorized based on severity into three groups- $\leq 4,5-10,>10$ and a comparison was made among CCALD, CALD, and asymptomatic groups (- Table 4).

Table 2 Clinical features of 19 symptomatic individuals

\begin{tabular}{|l|l|}
\hline Clinical symptoms & $\begin{array}{l}\text { No of affected } \\
\text { individuals (\%) }\end{array}$ \\
\hline Vision loss & $11(58)$ \\
\hline Hearing loss & $8(42)$ \\
\hline Seizures & $3(16)$ \\
\hline Headache & $6(32)$ \\
\hline Behavioral abnormality & $6(32)$ \\
\hline Neuroregression & $8(42)$ \\
\hline Gait abnormality & $9(47)$ \\
\hline $\begin{array}{l}\text { Bowel and bladder } \\
\text { abnormality }\end{array}$ & $2(11)$ \\
\hline Muscle weakness & $11(58)$ \\
\hline
\end{tabular}


Table 3 MRI findings of symptomatic and asymptomatic individuals

\begin{tabular}{|c|c|c|c|}
\hline \multirow{2}{*}{$\begin{array}{l}\text { White matter involvement: parieto- } \\
\text { occipital white matter }\end{array}$} & \multicolumn{3}{|c|}{ Disease presentation (no. of individuals) } \\
\hline & Symptomatic & Asymptomatic & Total \\
\hline Normal & 0 & 2 & 2 \\
\hline Abnormal & 19 & 1 & 20 \\
\hline \multirow{2}{*}{$\begin{array}{l}\text { White matter involvement: frontotemporal } \\
\text { white matter }\end{array}$} & \multicolumn{3}{|c|}{ Disease presentation } \\
\hline & Symptomatic & Asymptomatic & Total \\
\hline Normal & 10 & 3 & 13 \\
\hline Abnormal & 9 & 0 & 9 \\
\hline \multirow[t]{2}{*}{ Corpus callosum: splenium } & \multicolumn{3}{|c|}{ Disease presentation } \\
\hline & Symptomatic & Asymptomatic & Total \\
\hline Normal & 0 & 1 & 1 \\
\hline Abnormal & 19 & 2 & 21 \\
\hline \multirow[t]{2}{*}{ Corpus callosum: genu } & \multicolumn{3}{|c|}{ Disease presentation } \\
\hline & Symptomatic & Asymptomatic & Total \\
\hline Normal & 15 & 3 & 18 \\
\hline Abnormal & 4 & 0 & 4 \\
\hline \multirow[t]{2}{*}{ Corpus callosum: body } & \multicolumn{3}{|c|}{ Disease presentation } \\
\hline & Symptomatic & Asymptomatic & Total \\
\hline Normal & 17 & 3 & 20 \\
\hline Abnormal & 2 & 0 & 2 \\
\hline \multirow[t]{2}{*}{ Projection fibers: internal capsule } & \multicolumn{3}{|c|}{ Disease presentation } \\
\hline & Symptomatic & Asymptomatic & Total \\
\hline Normal & 12 & 3 & 15 \\
\hline Abnormal & 7 & 0 & 7 \\
\hline \multirow[t]{2}{*}{ Projection fibers: brain stem } & \multicolumn{3}{|c|}{ Disease presentation } \\
\hline & Symptomatic & Asymptomatic & Total \\
\hline Normal & 6 & 2 & 8 \\
\hline Abnormal & 13 & 1 & 14 \\
\hline \multirow[t]{2}{*}{ Cerebellum: white matter } & \multicolumn{3}{|c|}{ Disease presentation } \\
\hline & Symptomatic & Asymptomatic & Total \\
\hline Normal & 17 & 3 & 20 \\
\hline Abnormal & 2 & 0 & 2 \\
\hline \multirow[t]{2}{*}{ Cerebellum: atrophy } & \multicolumn{3}{|c|}{ Disease presentation } \\
\hline & Symptomatic & Asymptomatic & Total \\
\hline Absent & 15 & 3 & 18 \\
\hline Present & 4 & 0 & 4 \\
\hline \multirow[t]{2}{*}{ Basal ganglia } & \multicolumn{3}{|c|}{ Disease presentation } \\
\hline & Symptomatic & Asymptomatic & Total \\
\hline Normal & 14 & 3 & 17 \\
\hline Abnormal & 5 & 0 & 5 \\
\hline \multirow[t]{2}{*}{ Global atrophy } & Disease presen & & \\
\hline & Symptomatic & Asymptomatic & Total \\
\hline Absent & 9 & 3 & 12 \\
\hline Present & 10 & 0 & 10 \\
\hline Auditory pathway & Disease presen & & \\
\hline & Symptomatic & Asymptomatic & Total \\
\hline Normal & 7 & 3 & 10 \\
\hline Abnormal & 12 & 0 & 12 \\
\hline Visual pathway & Disease presen & & \\
\hline & Symptomatic & Asymptomatic & Total \\
\hline Normal & 2 & 1 & 3 \\
\hline Abnormal & 17 & 2 & 19 \\
\hline
\end{tabular}

Abbreviation: MRI, magnetic resonance imaging.

Indian Journal of Radiology and Imaging Vol. 31 No. 2/2021 $\quad$ c) 2021. Indian Radiological Association. 


\section{Discussion}

Approximately $60 \%$ of patients presenting with any form of X-ALD will eventually develop inflammatory form of cerebral disease. ${ }^{2}$ Prompt recognition of early cerebral involvement can facilitate timely intervention such as HSCT and this can have significant prognostic implications by arresting and interrupting the demyelination process. ${ }^{7}$ Neuroimaging can thus play a key role in early identification and decision making pertinent to treatment.

MRI signal changes in X-ALD occur due to inflammation and demyelination. The earliest T2 sequence abnormality is thought to be due to myelin membrane instability and oxidative stress that in turn triggers the initial damage that occurs in CALD. The contrast enhancement seen at later stages may be due to endothelial dysfunction as ABCD1 is thought to play a role in maintenance of tight junction. ${ }^{8}$ Histopathologically, $\mathrm{X}$-ALD is composed of three zones (Schaumburg zones): inner zone depicts irreversible gliosis and scarring, intermediate zone with active demyelination, and peripheral zone with active destruction. ${ }^{9}$

Typical neuroimaging findings of CCALD include confluent, symmetrical areas of T2 hyperintensity originating in the parieto-occipital periventricular white matter with early involvement of splenium of the corpus callosum and progresses in a posterior to anterior direction. ${ }^{10}$ The inner zone appears hypointense on T1W and markedly hyperintense on T2W, intermediate zone appears iso to hypointense on T2W MRI with postcontrast enhancement, and peripheral

Table 4 Predicted Loes score between symptomatic and asymptomatic individuals $(n=22)$

\begin{tabular}{|l|l|l|l|l|}
\hline \multirow{2}{*}{ Predicted Loes score } & \multicolumn{3}{|c|}{ Disease presentation } \\
\cline { 2 - 5 } & $\begin{array}{l}\text { CCALD } \\
(\boldsymbol{n}=10)\end{array}$ & $\begin{array}{l}\text { CALD } \\
(\boldsymbol{n}=9)\end{array}$ & $\begin{array}{l}\text { Asymptomatic } \\
(\boldsymbol{n}=3)\end{array}$ & $\begin{array}{l}\text { Total } \\
(\%)\end{array}$ \\
\hline$\leq 4$ & 0 & 0 & 3 & $3(13.6)$ \\
\hline $5-10$ & 8 & 5 & 0 & $13(59.1)$ \\
\hline$>10$ & 2 & 4 & 0 & $6(27.3)$ \\
\hline
\end{tabular}

Abbreviations: CALD, cerebral adrenoleukodystrophy; CCALD, childhood cerebral adrenoleukodystrophy.

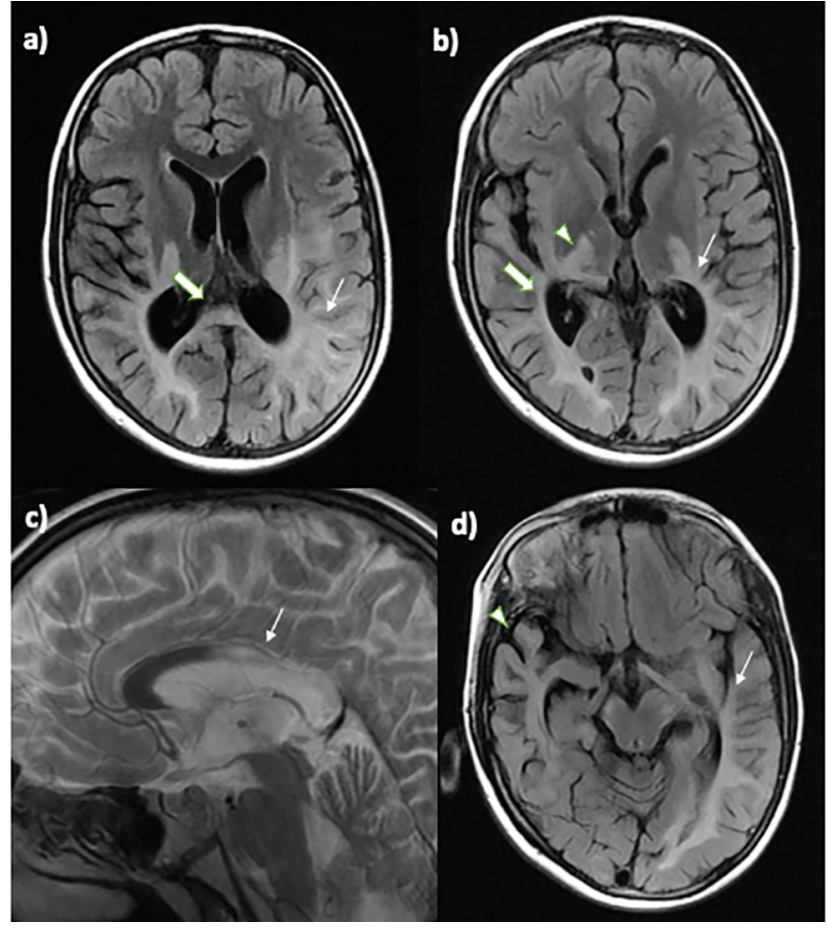

Fig. 1 Fluid-attenuated inversion recovery images of a 14-year-old symptomatic male: (A) Hyperintensity involving B/L parieto-occipital white matter (thin arrow) and splenium of corpus callosum (thick arrow); score-4. (B) Involvement of B/L lateral geniculate bodies (thin arrow), optic radiations (thick arrow), and B/L internal capsule (arrowhead); score-4. (C) Involvement of the body of corpus callosum (arrow); score-1. (D) Involvement of the white matter of B/L anterior temporal lobes (arrow) with atrophy of $\mathrm{B} / \mathrm{L}$ temporal lobes (arrowhead); score-3. Total Loes score-12.

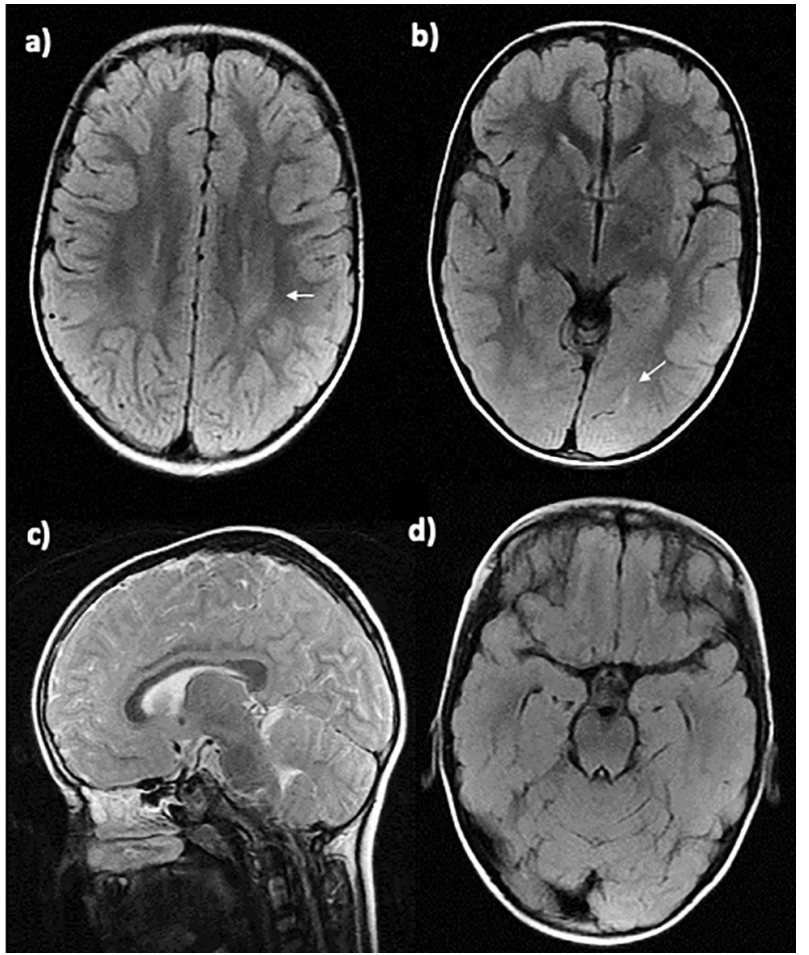

Fig. 2 Magnetic resonance images of a 8-year-old asymptomatic male: (A) Hyperintensity involving B/L parieto-occipital white matter (periventricular) on axial fluid-attenuated inversion recovery (FLAIR) images; score-1. (B) Foci of FLAIR hyperintensities involving the central part of $B / L$ parieto-occipital white matter (arrow); score-1. Sagittal T2 image (C) reveals normal corpus callosum. Axial FLAIR image at the level of brain stem (D) appears normal. Total Loes score-2 


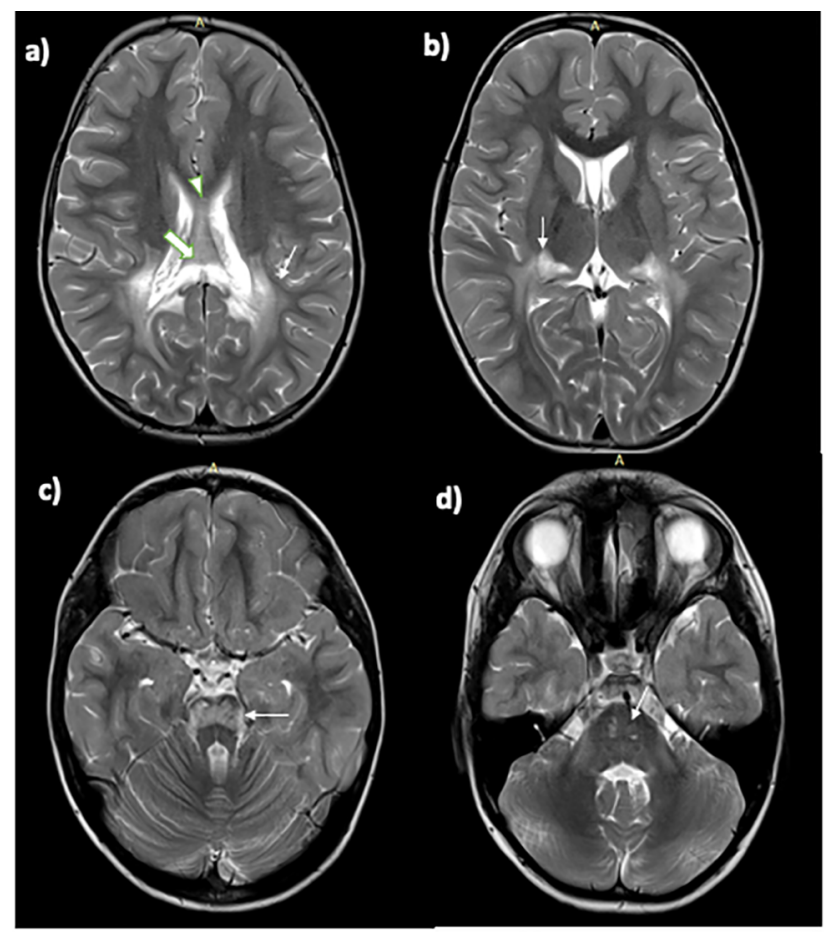

Fig. 3 Magnetic resonance images of a 9-year-old asymptomatic male: (A) T2 hyperintensity involving B/L parieto-occipital white matter (arrow), splenium (thick arrow) and genu (arrowhead) of corpus callosum; score-4. (B) T2 hyperintensity involving B/L optic pathways (arrow); score-2. T2 hyperintensities involving the brain stem (arrow in C) and pons (arrow in D); score-2. Involvement of B/L medial geniculate bodies (not shown in the images); score-1. Total Loes score-9.

zone appears mildly hyperintense on T2W MRI with no enhancement ( - Fig. 4). Postcontrast enhancement thereby indicates an advancing edge of active inflammation. In our series, we observed the classic picture of posterior predominance including the splenium of corpus callosum and parieto-occipital region in all the affected individuals. Consistent with prior reports, we observed that majority of these lesions in the brain originated in the midline of the corpus callosum, especially the splenium (100\%). ${ }^{11}$

Evaluation of conventional DWI and apparent diffusion coefficient map images will facilitate the detection of major diffusion abnormalities and identification of myelin edema in leukodystrophies that further strengthens the understanding of the pathogenesis. In DWI, the inner zone appears hypointense due to total loss of diffusional anisotropy, the intermediate inflammatory zone is moderately hyperintense indicating restricted water diffusion due to low-grade myelin edema and hypercellularity caused by lymphocytic infiltration (-Figs 4-6), and the most peripheral demyelinating zone is faintly hyperintense, perhaps due to T2-shine through. ${ }^{12}$ However, when the disease is advanced, the three characteristic zones were not evident on T2 images as well as DWIs (-Fig. 7).

Though conventional MRI is highly sensitive to detect white matter abnormalities, recent modalities like DTI seem to improve the sensitivity and specificity and it provides a more accurate identification and differentiation of such

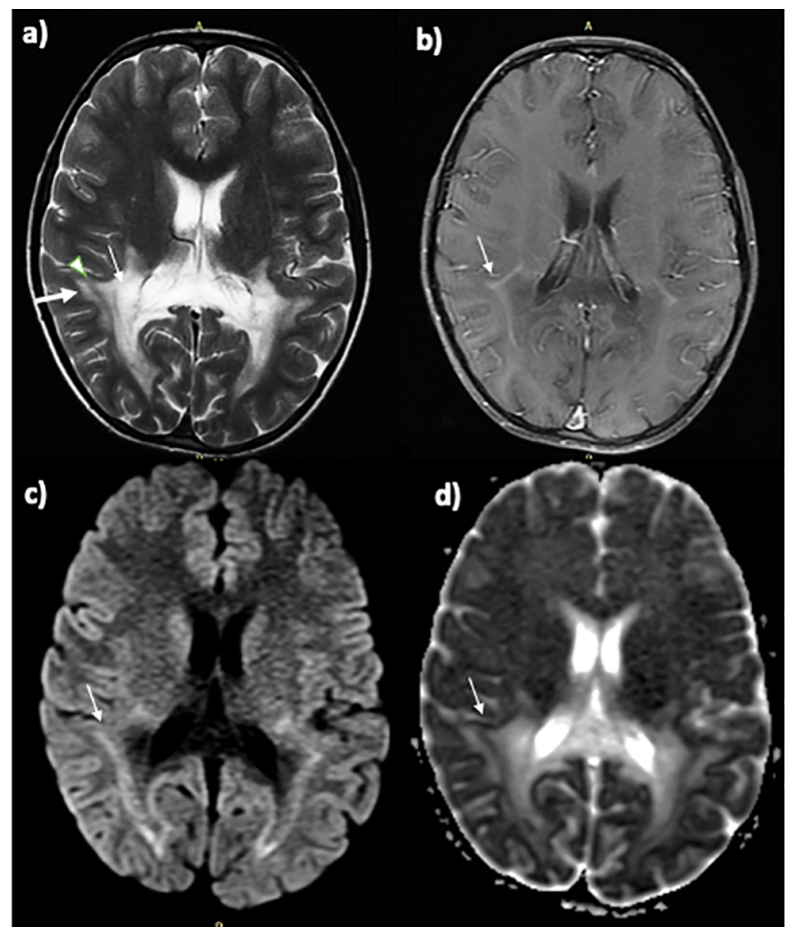

Fig. 4 Magnetic resonance images of a 12-year-old symptomatic male. (A) T2 image: Hyperintensity involving B/L parieto-occipital white matter and splenium of corpus callosum. Inner zone (thin white arrow) and peripheral zone (thick white arrow) are hyperintense. Intermediate zone (arrowhead) is isointense. (B) Postcontrast image: Enhancement (white arrow) of intermediate zone. (C) Diffusion-weighting imaging (DWI): Linear hyperintensity (arrow) in contrast-enhanced zone. (D) Apparent diffusion coefficient image: Linear hypointensity (white arrow) in areas of hyperintensity on DWI suggesting diffusion restriction. Total Loes score-14.

pathological processes in a very early stage. The anisotropic water diffusion in nerve fibers is the basis for the utilization of DTI to establish the nerve fiber pathways and such changes have been demonstrated in demyelinating diseases. ${ }^{13}$ The parameters employed in DTI include fractional anisotropy (FA), mean, radial, and axial diffusivity (MD, RD, and AD). The contribution to these indices comes from fiber arrangements, degree of myelination, and axonal integrity. Reduction in white matter FA reflects changes in tissue connectivity and integrity. Increase in $\mathrm{RD}$ and $\mathrm{AD}$ is related to damage in the myelin and axon, respectively. In a study by Ono et al, ${ }^{14}$ DTI parameters exhibited significant changes, despite the unaltered Loes score in early and very early stages of disease. They proposed that MD and RD measurements can potentially be used as an adjunct to Loes score in cases with very early stages of the disease.

Even in the setting of nonavailability of advanced techniques, employment of Loes score into clinical practice would still help the clinicians in follow-up and decision making on appropriateness of instituting HSCT. The improvement in survival has increased from 89 to $95 \%$ as compared with the survival probability of 45 to $54 \%$ prior to HSCT. Peter's et al suggested the patients with a Loes score $<9$ may benefit from $\mathrm{HSCT}^{6}$ and recently the threshold for inclusion of patients for HSCT with a Loes score of $<4$ has also been 


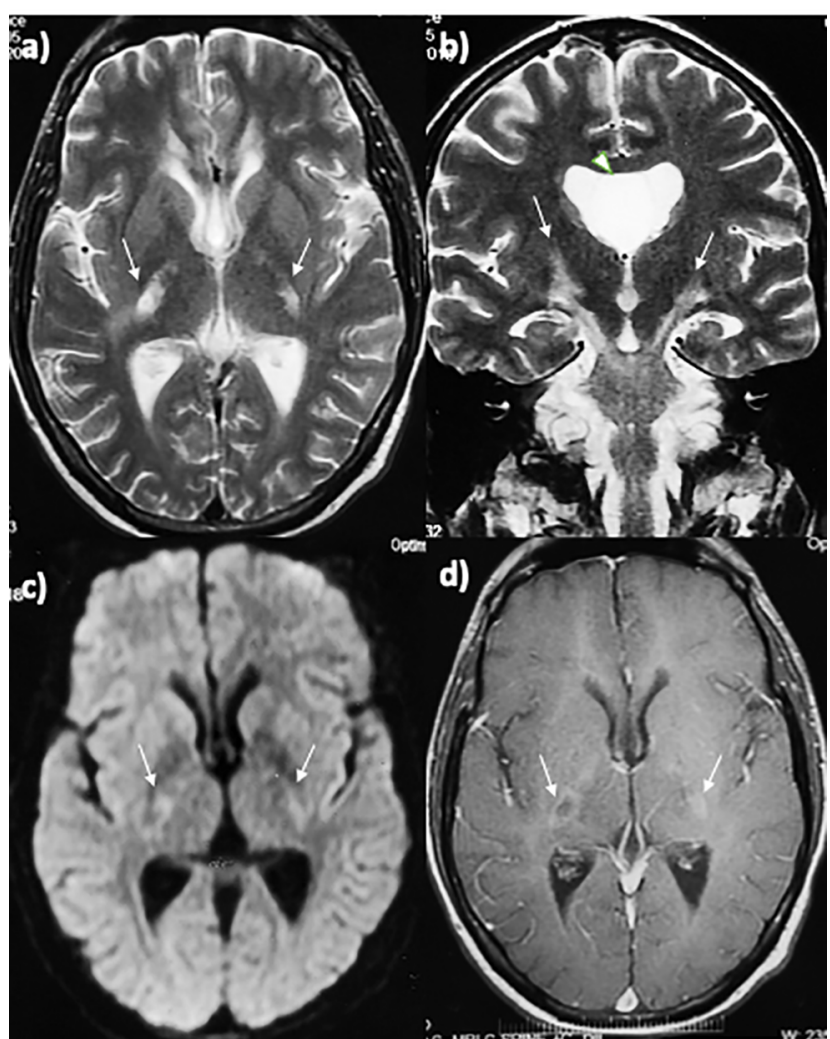

Fig. 5 Magnetic resonance images of a symptomatic male. (A) T2 image: Hyperintensity involving $B / L$ posterior limbs of internal capsule (arrows). (B) T2 image: Hyperintensity involving B/L corticospinal tracts (arrows). Incidental cavum septi pellucidi (arrowhead) seen. (C) Diffusion-weighting imaging (DWI): Rim of hyperintensity involving $\mathrm{B} / \mathrm{L}$ posterior limbs of internal capsule (arrows). (D) Postcontrast image: Rim of peripheral enhancement (arrows) in areas of hyperintensity on DWI.

proposed. ${ }^{15}$ Reports have also indicated that HSCT when performed in individuals with a high Loes score $(>10)$ had clinical and radiological progression of the disease. ${ }^{16}$ In our series, five individuals (26.3\%) had a Loes score of $<9$ and two unaffected individuals had a score of $<4$. These individuals would therefore be ideal candidates for HSCT.

In India, the current policy of funding of rare diseases through the Rastriya Arogya Nidhi in the context of the rare disease policy intends one-time funding for HSCT. This will likely benefit this population who cannot afford such modality of therapy. Individuals identified by cascade screening can have better outcomes as these individuals harboring neurological involvement without symptoms can be identified earlier and enrolled for treatment. Two of our asymptomatic individuals had a Loes score of $<4$ and are hence definite beneficiaries of the Rastriya Arogya Nidhi Scheme and efforts are being pursued for the same. ${ }^{17}$

The limitations of our study were that the neuropsychological tests that include evaluation of intelligence quotient (IQ) (full-scale IQ, verbal IQ, and performance IQ), five major cognitive domains (language, visuospatial skills, perception, visuomotor, or graphomotor skills, memory and attention or executive function), and adaptive skills were not assessed quantitatively. Hence, the categorization of asymptomatic
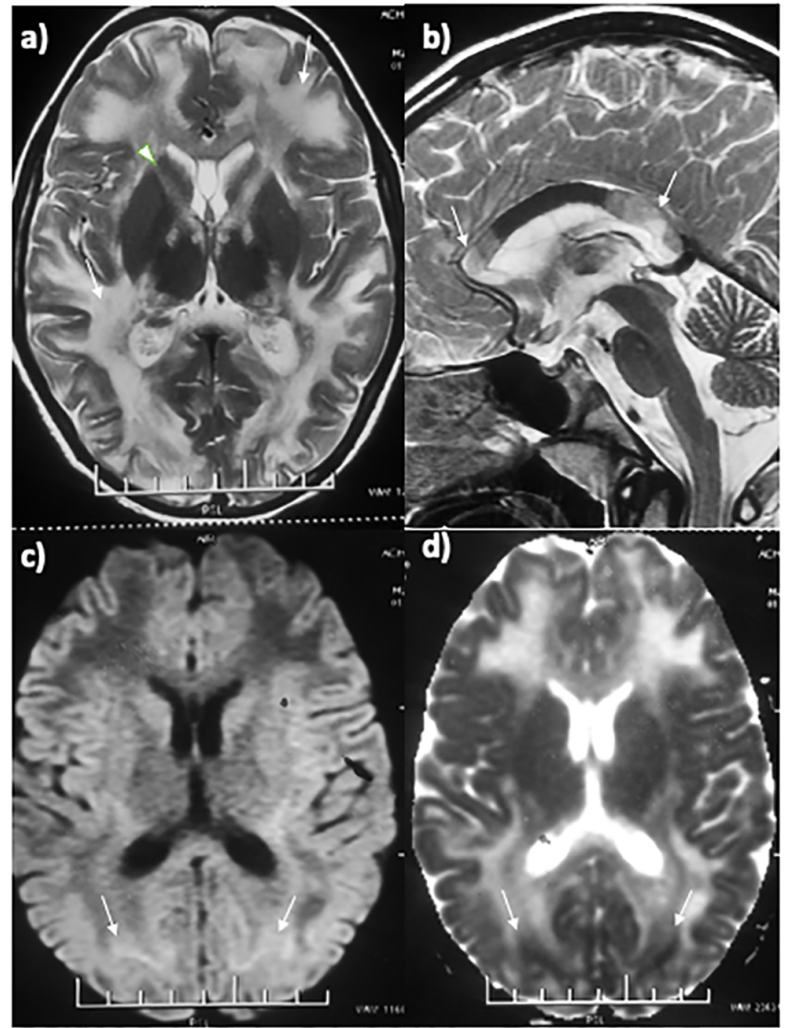

Fig. 6 Magnetic resonance images of symptomatic male with advanced disease. (A) T2 axial image: Hyperintensity involving $B / L$ frontal and parieto-occipital white matter (arrows) and B/L internal capsule (arrowhead). (B) T2 sagittal image: Hyperintensity involving the genu, splenium, and part of the body of corpus callosum (arrows). (C) Diffusion-weighting imaging (DWI): Linear subtle hyperintensity involving subcortical white matter of B/L occipital lobes (arrows). (D) Apparent diffusion coefficient image: Linear hypointensity (arrows) in areas of hyperintensity on DWI suggesting diffusion restriction. Total Loes score-22.

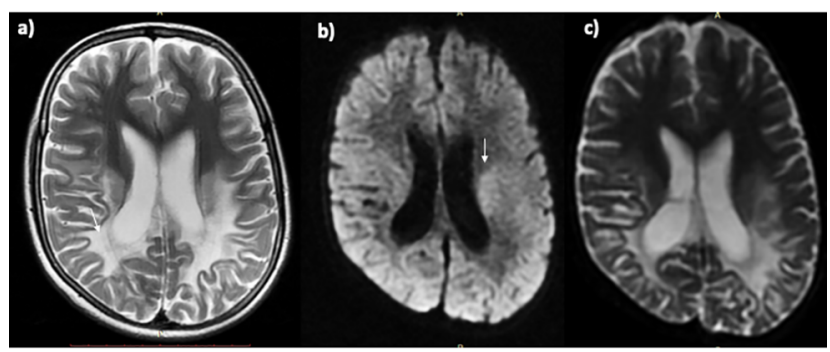

Fig. 7 Magnetic resonance images of a 14-year-old symptomatic male. (A) T2 image: Hyperintensity involving B/L parieto-occipital white matter (white arrow) with atrophy. The characteristic three zones of white matter changes are not evident. (B) Diffusion-weighting imaging (DWI): Linear hyperintensity is not evident in parieto-occipital white matter. Subtle hyperintensity is seen involving bilateral corona radiata (white arrow). (C) Apparent diffusion coefficient image: No loss of signal intensity corresponding to DWI hyperintensity suggesting $\mathrm{T} 2$ shine through.

individuals may not be genuinely representative. Also, serial MRIs in context of follow-up as designated were not performed due to issues in sedation and financial constraints. Though all scans were reviewed by a single radiologist, 
uniform MRI brain sections were not available. The subtle decrease in performance observed within some of the cognitive functional domains owing to axonal structural abnormalities may not be visible on conventional MRI. Further characterization of lesions using specialized imaging modalities such as magnetic resonance spectroscopy and DTI was not included in this analysis.

To conclude, our study represents the clinical and radiological profile for a large subgroup of individuals with X-ALD from India. We also demonstrated the utility of calculating the Loes severity score based on MRI. Employment of such scores in clinical practice would help in follow-up of the disease and in selecting the candidates for HSCT. This currently remains the only viable option for interrupting the demyelination process when performed at an early stage of the disease.

\section{Financial Support and Sponsorship}

All the authors confirm that the research was conducted in the absence of any academic funding or commercial funding that could be constructed as a potential support or grant for performing this study.

\section{Conflicts of Interest}

There are no conflicts of interest.

\section{References}

1 Moser HW, Raymond GV, Dubey P. Adrenoleukodystrophy: new approaches to a neurodegenerative disease. JAMA 2005;294(24):3131-3134

2 Wiesinger C, Eichler FS, Berger J. The genetic landscape of X-linked adrenoleukodystrophy: inheritance, mutations, modifier genes, and diagnosis. Appl Clin Genet 2015;8:109-121

3 Eichler F, Duncan C, Musolino PL, et al. Hematopoietic stem-cell gene therapy for cerebral adrenoleukodystrophy. N Engl J Med 2017;377(17):1630-1638

4 Kemper AR, Brosco J, Comeau AM, et al. Newborn screening for X-linked adrenoleukodystrophy: evidence summary and advisory committee recommendation. Genet Med 2017;19(1):121-126
5 Loes DJ, Hite $\mathrm{S}$, Moser $\mathrm{H}$, et al. Adrenoleukodystrophy: a scoring method for brain MR observations. AJNR Am J Neuroradiol 1994;15(9):1761-1766

6 Peters C, Charnas LR, Tan Y, et al. Cerebral X-linked adrenoleukodystrophy: the international hematopoietic cell transplantation experience from 1982 to 1999 Blood 2004;104(3):881-888

7 Suzuki Y, Isogai K, Teramoto T, et al. Bone marrow transplantation for the treatment of X-linked adrenoleukodystrophy. J Inherit Metab Dis 2000;23(5):453-458

8 Musolino PL, Gong Y, Snyder JM, et al. Brain endothelial dysfunction in cerebral adrenoleukodystrophy. Brain 2015;138(Pt 11) :3206-3220

$9 \mathrm{Kim} \mathrm{JH}$, Kim HJ. Childhood X-linked adrenoleukodystrophy: clinical-pathologic overview and MR imaging manifestations at initial evaluation and follow-up. Radiographics 2005;25(3):619-631

10 Liberato AP, Mallack EJ, Aziz-Bose R, et al. MRI brain lesions in asymptomatic boys with X-linked adrenoleukodystrophy. Neurology 2019;92(15):e1698-e1708

11 Loes DJ, Fatemi A, Melhem ER, et al. Analysis of MRI patterns aids prediction of progression in X-linked adrenoleukodystrophy. Neurology 2003;61(3):369-374

12 Patay Z. Diffusion-weighted MR imaging in leukodystrophies. Eur Radiol 2005;15(11):2284-2303

13 Filippi M, Cercignani M, Inglese M, Horsfield MA, Comi G. Diffusion tensor magnetic resonance imaging in multiple sclerosis. Neurology 2001;56(3):304-311

14 OnoSE, de Carvalho Neto A, Gasparetto EL, et al.X-linked adrenoleukodystrophy: correlation between Loes score and diffusion tensor imaging parameters. Radiol Bras 2014;47(6):342-349

15 Warren DJ, Connolly DJA, Wilkinson ID, Sharrard MJ, Griffiths PD. Magnetic resonance spectroscopy changes following haemopoietic stem cell transplantation in children with cerebral adrenoleukodystrophy. Dev Med Child Neurol 2007;49(2):135-139

16 Bladowska J, Kulej D, Biel A, et al. The role of MR imaging in the assessment of clinical outcomes in children with X-linked adrenoleukodystrophy after allogeneic haematopoietic stem cell transplantation. Pol J Radiol 2015;80:181-190

17 Main.mohfw.gov.in. 2020 [cited 29 November 2020]. Available from: https://main.mohfw.gov.in/sites/default/files/RAN Guideline_2019.pdf. Accessed July 21, 2020 